\title{
Spatially Resolved Soft X-ray Spectroscopy in Scanning X-ray Microscopes
}

Adam P. Hitchcock, ${ }^{1 *}$ Peter Krüger, ${ }^{2}$ Carla Bittencourt, ${ }^{3}$ George D.W. Swerhone ${ }^{4}$ and John R. Lawrence $^{4}$

1. Chemistry \& Chemical Biology, McMaster University, Hamilton, ON, Canada.

2. Graduate School of Science and Engineering, Chiba University, Chiba, Japan.

3. Chemistry, University of Mons, Mons, Belgium.

4. Environment and Climate Change Canada, Saskatoon, SK, Canada.

* Corresponding author: aph@mcmaster.ca

Near edge X-ray absorption fine structure (NEXAFS) spectroscopy is a powerful probe of the structure and bonding of materials, especially in the soft X-ray region $(50-2500 \mathrm{eV})$ where the high experimental energy resolution and small natural linewidths provide much better chemical sensitivity than hard X-ray spectroscopy. NEXAFS measured in soft X-ray scanning transmission X-ray microscopes (STXM) [1] is a powerful tool for materials and environmental analysis. Together STXM-NEXAFS can provide chemical speciation and quantitative mapping in 2D and 3D, with a spatial resolution better than $30 \mathrm{~nm}$. In the last few years the spatial resolution has been dramatically improved from $\sim 30 \mathrm{~nm}$ to less than $5 \mathrm{~nm}$ using ptychographic coherent diffraction imaging $[2,3]$. When the synchrotron source illuminating a STXM microscope is an elliptically polarizing undulator, site specific geometric and electronic anisotropy and magnetic properties can be probed and mapped. Detection of X-ray fluorescence (XRF) signal while scanning the incident photon energy provides greatly enhanced NEXAFS detection limits [4]. Results from recent studies of linear dichroism in boron nitride nanostructures [5], circular dichroism of individual magnetosomes in magnetotactic bacteria [2] and XRF-based metal speciation in an environmental biofilm [6] are used to illustrate these capabilities.

Figure 1 presents images and linear polarization dependent B 1s spectra of a BN nanoribbon $(\sim 100 \times 200 \mathrm{~nm}$ $\mathrm{x} 10 \mathrm{~nm}$ ). When the shape and dichroic response of the B 1s and $\mathrm{N} 1 \mathrm{~s}$ spectra were compared with high level VASP calculations (explicit core hole, core excited unit cell at centre of a 4x4x1 super-cell) the detailed layer stacking was deduced [5]. B 1s and N 1s spectromicroscopy of bamboo and nanotube BN structures are being used to investigate their electronic and spatial structure.

A river biofilm was exposed to $10 \mu \mathrm{g} / \mathrm{mL} \mathrm{Ni}(\mathrm{II})$ for 5 days to examine chemical states and possible preferential absorption of the toxic Ni ions. NEXAFS stacks at the $\mathrm{Ca}, \mathrm{N}, \mathrm{O}, \mathrm{Ni}, \mathrm{Al}, \mathrm{Si}$ and $\mathrm{Fe}$ edges were measured and analyzed to generate elemental maps and $\mathrm{Mn}, \mathrm{Ni}$ and $\mathrm{Fe}$ speciation (Fig. 2). As found in earlier studies [7] the Ni was found collocated with Mn and Fe, reinforcing the conclusion that metal ion metal ion interactions are important in determining the fate of $\mathrm{Ni}$ (II) in the environment. Another river biofilm sample, exposed to $1 \mu \mathrm{g} / \mathrm{mL} \mathrm{Ni(II)} \mathrm{for} 24$ hours, was examined with both transmission and XRF detection [6]. In this case the higher XRF sensitivity allowed identification of $\mathrm{Ni}(\mathrm{III})$ as well as $\mathrm{Ni}$ (II) inside and outside a cyanobacterium (Fig. 3) [8].

\section{References:}

[1] AP Hitchcock in "Handbook on Nanoscopy" eds. G. Van Tendeloo, D. Van Dyck and S. J. Pennycook (Wiley).

[2] XH Zhu et al., Proc. Nat. Acad. Sci. 113 (2016), p. E8219.

[3] Y Yu et al., Nature Communications 9 (2018), p. 921.

[4] AP Hitchcock et al., Env. Sci. Tech. 46 (2012), p. 2821.

[5] P Krüger et al., Rad. Phys. \& Chem (2019) in press. doi: 10.1016/j.radphyschem.2019.01.014 
[6] JR Lawrence et al., J. Microscopy 261 (2016), p. 130.

[7] A.P. Hitchcock et al., Geobiology 7 (2009), p. 432.

[8] Research performed using the ambient-STXM on beamline 10ID1 at the Canadian Light Source, which is supported by the Canada Foundation for Innovation.

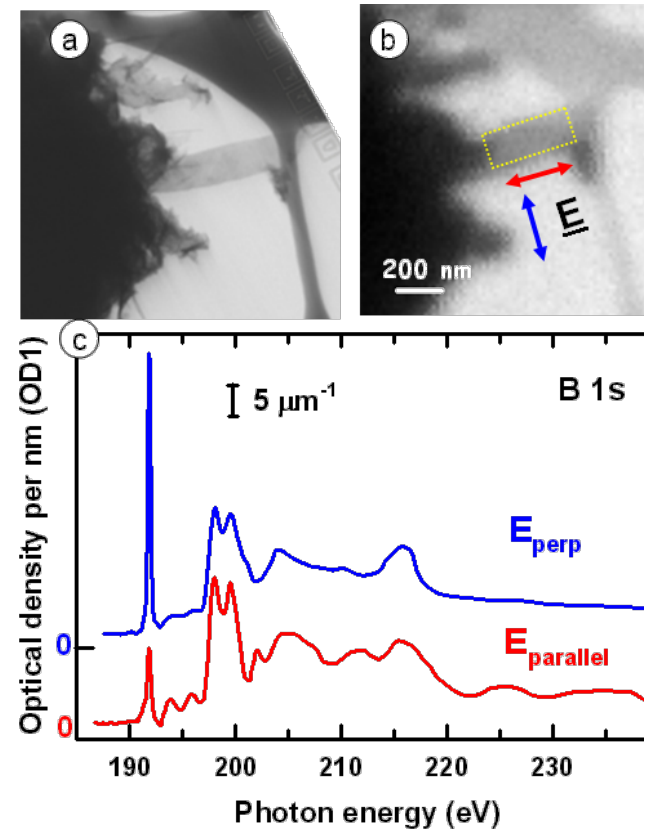

Figure 1. (a) Transmission electron microscope (TEM) image in same region as (b) STXM transmission image at $191.9 \mathrm{eV}$ of a $\mathrm{BN}$ nanoribbon (yellow box). (c) B 1s NEXAFS spectra of the nanoribbon with $\mathrm{E}_{\perp}$ and $\mathrm{E} / /$.
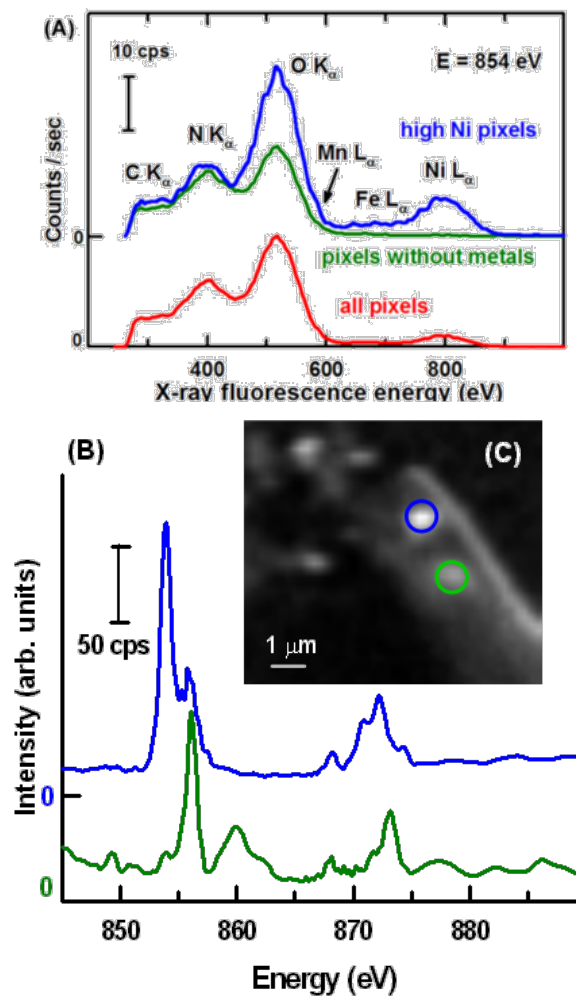
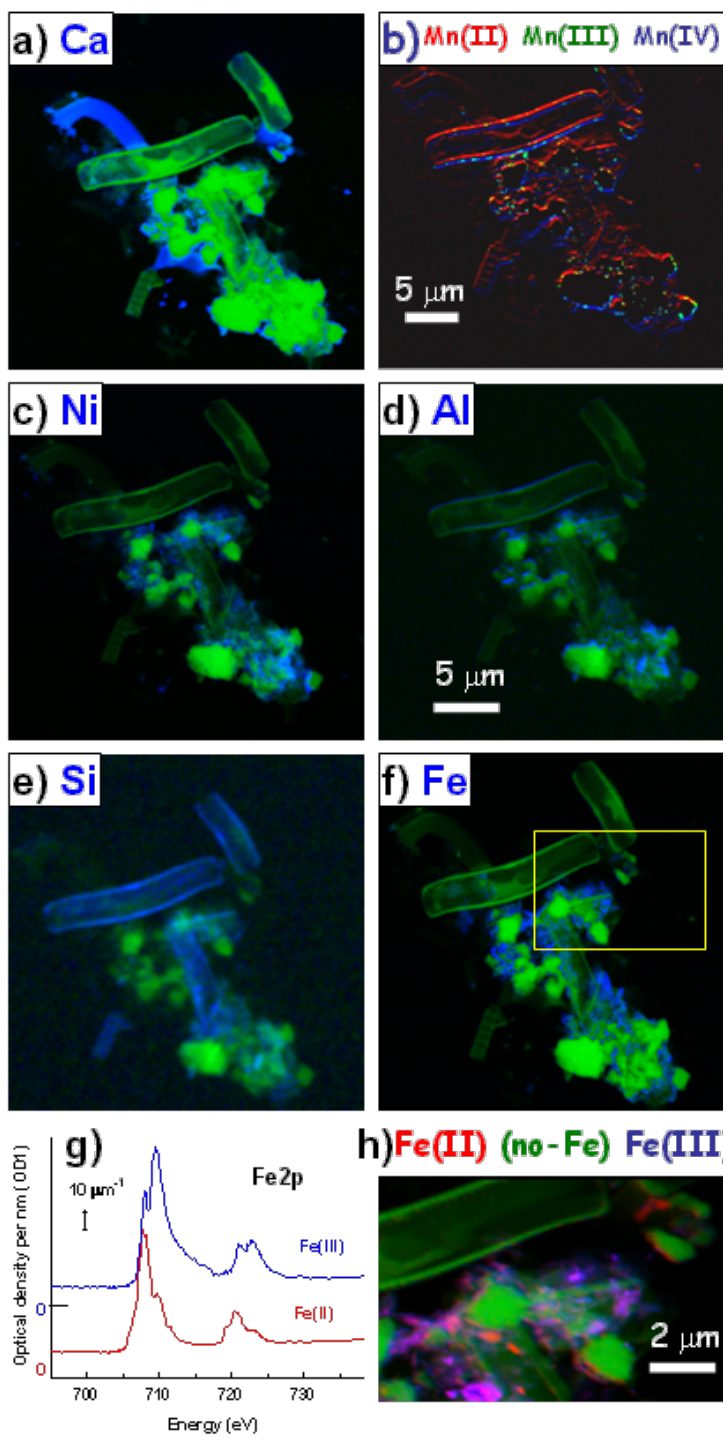

h) $\mathrm{Fe}$ (II) (no-Fe) $\mathrm{Fe}$ (III

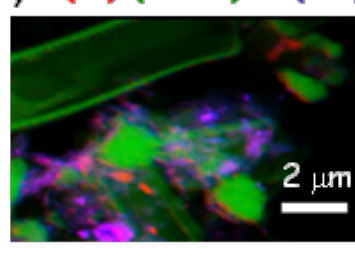

Figure 2. (a-f) Elemental maps of the indicated elements (blue) from peak - pre-edge signals, combined with pre-edge images (green) from a river biofilm exposed to $10 \mu \mathrm{g} / \mathrm{mL} \mathrm{Ni}$ (II) for 5 days. (g) Fe L NEXAFS of Fe(II) and Fe(III) used to fit the Fe L stack of the biofilm. (h) Fe speciation maps of yellow box area in (f).

Figure 3. (A) XRF spectrum measured with primary energy of $854 \mathrm{eV}$ from several areas of a river biofilm exposed to $1 \mu \mathrm{g} / \mathrm{mL} \mathrm{Ni}(\mathrm{II})$ for 1 day. (B) Ni L NEXAFS spectra of the spots circled with blue and green in (C) average XRF yield image (60 energies from 845 to $890 \mathrm{eV}$. 